\title{
Marketing en tiempos de crisis generado por la COVID-19
}

\section{Marketing in times of crisis generated by COVID-19}

LABRADOR, Hender E. ${ }^{1}$

SUAREZ, Jhon J. ${ }^{2}$

SUAREZ, SIR A. ${ }^{3}$

\section{Resumen}

La pandemia COVID-19 ha tenido un impacto sin precedentes a nivel mundial, afectando a todos los sectores. El comportamiento de los consumidores ha sufrido un cambio apresurado, motivado al cierre de las industrias y negocios, así como al confinamiento obligatorio de la población; esto produjo un volcamiento masivo hacia el Ecommerce. Se realiza un análisis descriptivo del Inbound Marketing como estrategia a seguir en la crisis generada por la COVID-19.

Palabras clave: comercio electrónico, inbound marketing, COVID-19.

\begin{abstract}
The COVID-19 pandemic has had an unprecedented impact globally. Affecting all sectors; suffering an impact on the behavior of consumers, having a hasty change, motivated by the closure of industries and businesses, as well as the mandatory confinement of the population; this produced a massive turn towards Ecommerce. A descriptive analysis of Inbound Marketing is carried out as a strategy to follow in the crisis generated by COVID-19.

Key words: ecommerce, inbound marketing, COVID-19.
\end{abstract}

\section{Introducción}

Sin duda alguna el 2020 ha sido un año que está revolucionando la forma en la que vivimos, nos comunicamos y principalmente como compramos, teniendo un impacto fundamental en el marketing que ha dado un giro de 360 grados para poder satisfacer rápidamente las necesidades de una población obligada al confinamiento. La situación causada por la expansión de la COVID-19 alrededor del mundo ha puesto en jaque la economía de la mayoría de los países, obligando al comercio a cerrar sus puertas y a replantearse la manera de como relacionarse con sus clientes y es que la COVID-19 desencadenó un tsunami de innovación (Morales, 2020), congeló industrias enteras y dejó arcaicas aquellas estrategias cuidadosamente planificadas por especialistas, viéndose obligados a implementar campañas acorde a la crisis, convirtiéndose en un desafío extraordinario (Hill, 2020).

El encuentro inicial con la pandemia fue, y aún es, durísimo (Hugo, 2020). Estamos migrando hacia una nueva normalidad y, con este cambio, aún inseguro tanto en su impacto y duración, llega la convicción de que buscar una nueva forma de actividades comerciales es una cuestión de supervivencia para las empresas; ya estábamos

\footnotetext{
${ }^{1}$ Docente. Facultad de Ciencias Económicas y Sociales. Universidad Católica del Táchira, Venezuela. Correo electrónico: hender.labrador@ucat.edu.ve

${ }^{2}$ Docente. Áreas básicas. Centro Educativo Rural La Primavera, Colombia. Correo electrónico: Jjsuarez8@gmail.com

${ }^{3}$ Docente. Programa Ingeniería de Sistemas. Universidad Francisco de Paula Santander Ocaña. Grupo de Investigación Grucite. Correo electrónico: sasuarezc@ufpso.edu.co
} 
viviendo un cambio en cómo las empresas manejaban sus negocios debido a la digitalización y con el impacto causado por pandemia mundial de la COVID-19, esos procesos fueron bruscamente acelerados y muchos paradigmas transformados, expresando que una reinvención es necesaria, lo que obliga a empresas y consumidores a buscar nuevas formas de hacer negocios (Gutzwiller, Dunz-Real, y Reinsurance, 2020) como Ebusiness o B2B (Shelly, 2010), y que se hacen la interrogante; ¿El Marketing Digital, es el camino correcto para enfrentar este tiempo desafiante?, Incluso los publicistas más expertos, acostumbrados a aplicar estrategias y creatividades rápidamente, se cuestionan, en ¿cómo afrontar este escenario sin precedentes?.

En este contexto Kaminsky (2004) advierte que "las crisis no son un fenómeno nuevo y, tanto las causas como las consecuencias sugieren la existencia de variedad". "Ello hace que en cada uno de estos períodos, los profesionales de marketing se encuentren en territorio poco conocido, porque no hay dos recesiones que sean iguales" (Quelch y Jocz, 2009).

Las pequeñas y medianas empresas, son responsables por el $80 \%$ de los empleos formales con una caja para operar en un lapso de 30 a 120 días. Eso hace aún más desafiante pasar la crisis sin fallar. Las grandes empresas, por su tamaño, escala, reconocimiento de marca y capacidad de caja, tienen mayores condiciones de pasar por la crisis sin grandes impactos a mediano y largo plazo. Sin embargo con la COVID-19 nos vimos obligados hacer cambios en las empresas, adaptarnos al confinamiento, trabajar desde casa, ver despidos y quiebras. Todo se ha visto afectado y hemos encontrado nuevas formas de vivir y resolver los problemas del día a día.

Donde las economías más débiles que aún estaban en proceso de crecimiento, (como Colombia), el reto es aún más grande, debido a que las pequeñas y medianas empresas (PYMEs) generan aproximadamente el 67-80\% de los empleos formales en Colombia (DANE, 2018).

En este escenario generado por la pandemia, estas empresas afrontan numerosos desafíos, como: mejorar el manejo del flujo de caja, migrar el modelo de negocio a digital, y sobretodo, transformar el departamento de Marketing, que muchas veces es subestimado solamente como un costo, en lugar de un centro de adquisición de ingresos de manera previsible, escalable y sostenible.

El mercado nunca volverá hacer el mismo, menos la forma de conquistar clientes. Ahora, quedarse atrás no es una opción y los contextos en los que vivimos demanda una adaptación y reacción inmediata, cambiando el enfoque para relacionarse y generar contenido de productividad y home office. Pues cada día vivimos un nuevo escenario; donde empresas que manejaban un marketing tradicional ya se están adaptando al nuevo escenario, como:

- Instituciones educativas reemplazando las clases presenciales por clases y cursos online o con alternancia.

- Profesionales independientes ofreciendo consultoría a distancia.

- Tiendas físicas explorando las redes sociales y transfigurando su modelo de negocio.

Por otro lado las empresas que ya manejan estrategias de Marketing Digital, como el Inbound Marketing (Meijomil, 2020) y el Win-Win (Gómez, 2020) están al frente en este escenario.

"Las reglas del marketing han cambiado y su negocio puede beneficiarse de este cambio. El Inbound Marketing le muestra cómo ser encontrado por más prospectos que ya están buscando lo que tiene para vender" (Halligan y Shah, 2009).

Indudablemente es hora de replantearse, cuál es el papel que juega el marketing dentro de nuestra empresa, tanto para confrontar la crisis como para emerger fortalecidos después del período negativo (Toledo y Armas, 2020). Para esto debemos hacernos algunas interrogantes: ¿Cómo adaptarse para sobrevivir a la tormenta, para 
luego reconstruir?; ¿Qué acciones tomar para continuar vendiendo en este período difícil?; ¿Por qué el Inbound Marketing es ideal para el momento?

\section{Metodología}

El método descriptivo es uno de los métodos cualitativos que tienen como objetivo la evaluación de algunas características de una población o situación en particular. En la investigación descriptiva, el objetivo es describir el comportamiento o estado de un número de variables.

Según Hernández Sampieri (2018, p. 60), "los estudios descriptivos permiten detallar situaciones y eventos, es decir cómo es y cómo se manifiesta determinado fenómeno y busca especificar propiedades importantes de personas, grupos, comunidades o cualquier otro fenómeno que sea sometido a análisis".

La COVID-19 es sólo el principio de una evolución significativa en las creencias y actitudes. Este cambio acelerará una serie de cambios estratégicos en las empresas y consumidores, con el miedo ahora convirtiéndose en el estado emocional emergente. Los sucesos actuales están precipitando este cambio de mentalidad, mostrándonos las acciones durante y después de la actual pandemia. Los consumidores no solo se enfrentaran al temor del bienestar y su salud, de igual forma tendrán preocupaciones sobre la incertidumbre financiera que ha brotado a causa de las empresas que se ven obligadas a frenar labores.

\section{Resultados}

En tiempos de crisis, el Marketing es una de las áreas más impactadas dentro de cualquier empresa. Por eso, es muy importante probar el valor generado por las estrategias de marketing, es necesario medir los resultados de las estrategias realizadas, para saber si las medidas tomadas de hecho están funcionando. Con el análisis, se entenderán que campañas están trayendo resultados más expresivos, así se podrá invertir más y mejorar los resultados. Además de examinar los resultados, es fundamental entender lo que funciona en cada época del año o incluso en la misma crisis

\section{1. ¿Cómo afecta la COVID-19 el mercado y los consumidores?}

El coronavirus ha traído consigo una serie de consecuencias económicas debido a las restricciones de movilidad, el confinamiento y las limitaciones globales a las que se ha visto expuesto el mercado. Durante la primera etapa de la pandemia hubo un salto de 14 puntos en el número de adultos intranquilizados por el impacto económico del coronavirus, con un $69 \%$ diciendo que están preocupados, en una encuesta de Morning Consult realizada del 24 al 26 de febrero de 2020 (Famanía, 2020), en comparación con la quincena anterior. Dejando ver que los métodos tradicionales de Marketing y Ventas ya no eran suficientes y significaba que las empresas debían trabajar para reinventar estrategias de marketing y mejorar la sensación de seguridad, bienestar y que promovieran la calma de los consumidores, que se vieron obligados a trabajar desde casa, generando un cambio en la zona de confort de la vida administrativa y profesional de las personas, haciendo que se desplazaran cada vez más hacia lo virtual.

\subsection{Canales y estrategias}

Antes de la pandemia, considerables empresas estaban cerrando el primer semestre con sus estrategias de evolución y retención ya comprobadas siendo puestas en práctica, con presupuestos destinados a estrategias enfocadas en ventas, que durante la crisis no se están desempeñando bien ni generando los resultados esperados. El marketing de los sitios web de las empresas se está derrumbando para la mayoría de los segmentos (excepto medios, farmacéutica, finanzas, alimentación y salud) donde los presupuestos fueron cortados o re destinados (ver Figura 1). 
Figura 1

Tráfico Web en segmentos de la industria

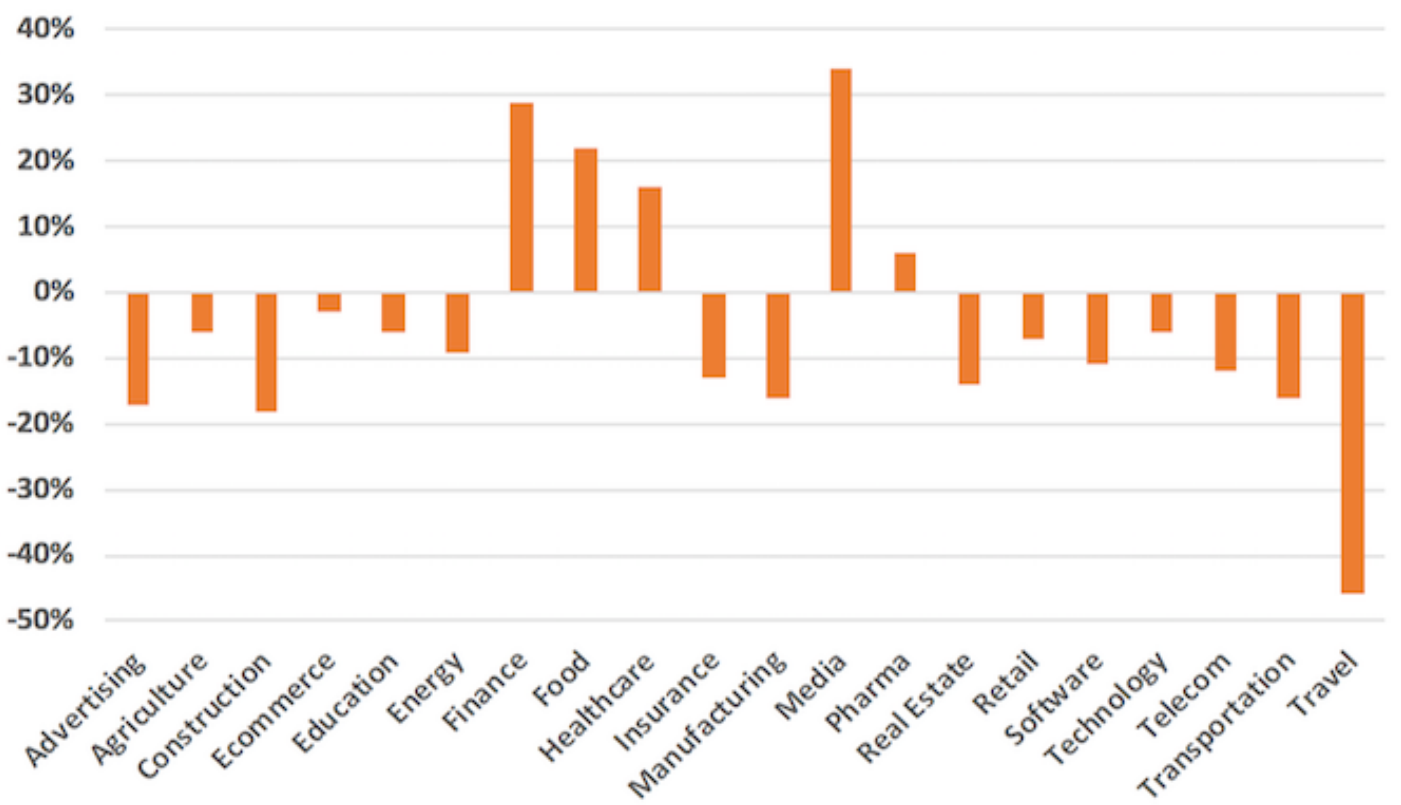

Fuente: Schüler (2020)

Actualmente estamos viviendo la segunda fase del confinamiento, donde progresivamente, algunos sectores de la economía han reactivado paulatinamente sus actividades. Para hacerlo debidamente es obligatorio acatar no sólo las recomendaciones de los entes de control, sino analizar nuestros planes estratégicos.

En corto tiempo, medidas de seguridad y salud pública tuvieron un impacto directo en la mayoría de los segmentos de negocios. Diversas empresas, principalmente PYMEs que tenían sus operaciones físicas, comenzaron a tener sus acciones tradicionales inviables. Eso se reflejó un declive apresurado en las ventas, dejando esos negocios en un peligro inminente.

Donde las empresas que ya utilizan estrategias de Marketing Digital para relacionarse y vender, como el Inbound Marketing, están al frente en este escenario.

Según Samsig (2019), el Inbound Marketing es "una estrategia que se basa en atraer clientes con contenido útil y relevante, agregando valor en cada una de las etapas del recorrido del comprador." Por otra parte la empresa de software Marketo (Valdés, 2019), define el Inbound Marketing cómo "una estrategia que utiliza muchas formas de marketing improvisado (marketing de contenido, blogs, eventos, SEO, redes sociales y más) para crear conciencia de marca y atraer nuevos negocios. En contraste con el marketing saliente, donde los mercadólogos intentan encontrar clientes, el marketing entrante llama la atención de los clientes y hace que la empresa sea fácil de encontrar" (ver Figura 2). 
Figura 2

Claves del Inbound Marketing

\section{Inbound Marketing}

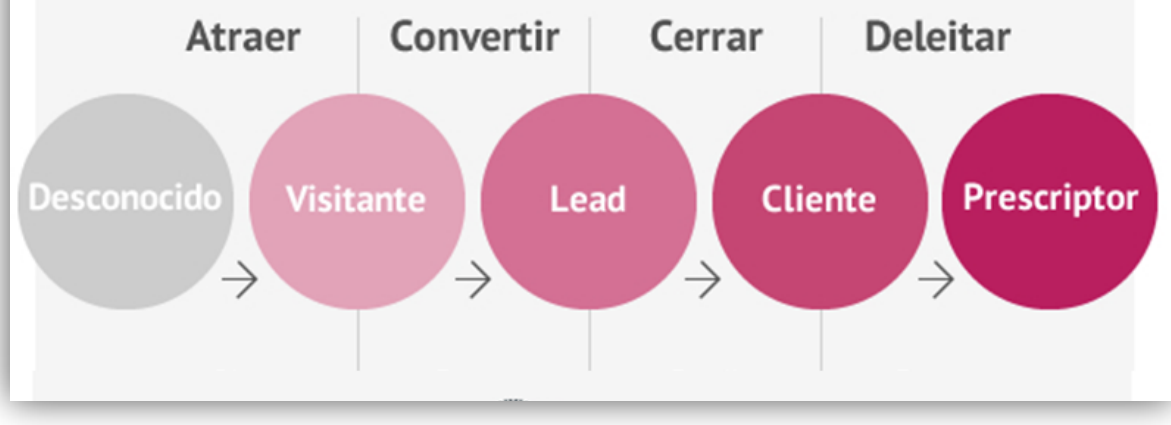

Fuente: Fernández (2013)

\subsection{Implementación del Inbound Marketing}

Ante alarmas de recesión y crisis económica, diversas dudas y riesgos navegan la mente de los emprendedores, empresas y profesionales de marketing, como: ¿Qué puedo hacer para continuar posicionado en este momento difícil?, ¿Cómo retengo mis clientes? ¿Cómo me posiciono? ¿Dónde debo invertir lo que tengo?, ¿Debo seguir con campañas físicas tradicionales?, ¿Cómo compruebo que las estrategias de marketing están generando los resultados esperados?, Esas son dudas y retos que cualquier negocio podría tener a lo largo de su existencia, pero el escenario hoy es muy diferente del habitual gracias al COVID19.

Lo que hace conveniente reconsiderar las acciones de marketing que vamos a realizar. Es por este motivo que utilizar las herramientas de Inbound Marketing puede resultar una excelente alternativa para lograr nuestros objetivos en un corto plazo y con bajo presupuesto, aunque el camino no es fácil, tiene más garantías de éxito que seguir con las estrategias del pasado.

Es hora de reinventar una estrategia que permita seguir compitiendo dentro de un mercado tan inestable a causa de la pandemia.

El Inbound Marketing, está al frente en este escenario, permitiendo a las empresas adaptarse al momento y ampliar ese frente de adquisición de clientes, realizando de forma más rápida las estrategias necesarias que les permita seguir generando demanda a un corto y largo plazo, recuperándose con más facilidad luego de la crisis y volver a una etapa de crecimiento.

Las estrategias basadas en inbound marketing (ver Figura 3) proporcionan ventajas a las empresas que lo ponen en práctica. Algunas de ellas son:

- Aumenta los contactos cualificados de marketing (MQL): los multiplica por 7,3 en un año y por 9,8 en dos años.

- Aumenta los registros (leads): los multiplica por 3,8 en un año y por 14,7 en dos años.

- Incrementa las visitas que recibe la página web: las multiplica por 4,7 el primer año y por 24,3 en dos años. 
Figura 3

Ventajas del inbound marketing
MQL

Crecimiento acumulado
Registros

Crecimiento acumulado
Visitas

Crecimiento acumulado

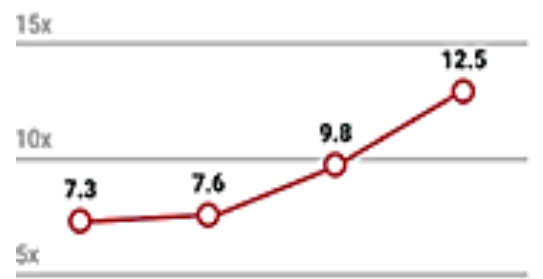

$2 x$

Semestrez Semestres Semestred Semestres

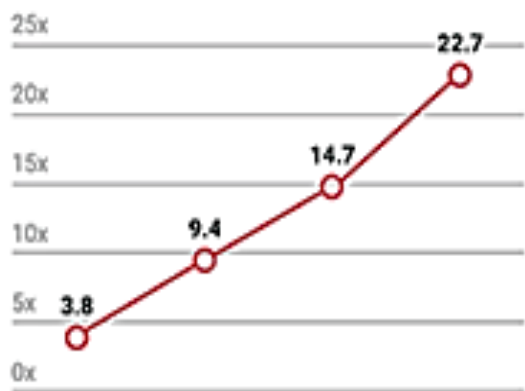

Semestre 2 Semestre 3 Semettre4 Semestres

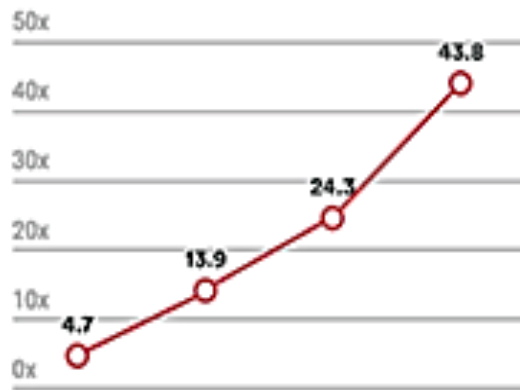

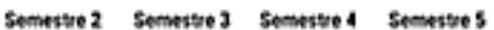

Fuente: Valdes (2019)

El Inbound Marketing centra sus esfuerzos en satisfacer la demanda de información del cliente,.Es por ello, que el contenido de email no debe ser un escaparate hacia fuera sino toda una llamada de atención hacia el siguiente paso. Es ahora cuando una comunicación personalizada y directa con los clientes, confinados en casa, puede traer grandes beneficios en situaciones de crisis como la que ha provocado la COVID-19.

El envío de mails con información atractiva sobre la contexto actual de las empresas (Martínez, 2000), servicios disponibles o las ofertas comerciales derivadas del momento, está reflejando ser una de las técnicas más apropiadas para mantener una dependencia con el cliente, decisiva en las fases del Inbound para atraer y fidelizar. Las estadísticas de apertura de emails, manifiestan su efectividad en tiempos de crisis como la actual incitada por la COVID-19.

Los usuarios durante esta época de pandemia están muy receptivos de cualquier información proveniente de sus proveedores y negocios percibidos en las redes sociales. Por ello la apertura de emails y los clics en enlaces publicitarios superan las tasas a otros envíos anteriores con información comercial. Pero para rentabilizar el envío de estos correos, deben estar bien elaborados y expresar una serie de requisitos fundamentales que los conviertan en parte esencial de la estrategia de Inbound Marketing.

Algunos de los tics utilizados por los profesionales del Inbound Marketing (Valdés, 2019), son:

- Interacción en redes sociales y otros medios de comunicación para conocer el mercado que más le interesa a los usuarios y así poder dar respuesta de forma personalizada a través del email.

- Conciben contenidos de interés que generan la curiosidad y provocan la interacción de los usuarios, ya sea a través de la web o de compartir el contenido.

- Colocar enlaces en la web y redes sociales, para dar a conocer los productos y servicios.

- Utilizan las landing page, elaborada especialmente para la situación de crisis que se presente, dejando claro la disponibilidad de los productos y servicios.

- Segmentan campañas de email, adaptando el mensaje en función de las empresas, recompensando interacción de los usuarios con cupones de descuento, regalos u ofertas exclusivas. 


\subsection{Colaboración en el núcleo de la estrategia de marketing}

El Inbound Marketing junto con el Win-win se está convirtiendo en un nuevo estándar para el mundo de los negocios. El Win- Win no es más que una estrategia de marketing que traducida del inglés, significa Ganar-Ganar, que tiene como objetivo que todas las partes salgan beneficiadas (empresas, distribuidores, y consumidores). Según Jaime Cavero (2019), Inversor de Startups y Presidente de la MentorDay "win-win, se trata de una de las estrategias de negociación más efectivas ante la realidad que enfrentamos".

Enmarcado en lo anterior, El área de marketing de las empresas están incrementando las campañas que van dirigidas al cliente final, donde el empresario y el canal de distribución van de la mano.

En una época de crisis donde todos quieren ganar, los profesionales del marketing utilizan su producto como una opción ganadora para posicionarse como un partner, utilizando propuestas de valor centradas en proporcionarle al cliente, su apoyo como profesional de forma continuada. Los clientes valoran muy positivamente el acceso a información y documentación útil, el asesoramiento. En definitiva, esta época es una grandiosa oportunidad para edificar una relación de colaboración continuada en el tiempo, llena de sentido y contenidos.

Debido a la situación que el mundo está viviendo a causa de la COVID-19 y el confinamiento obligado, las Redes Sociales se convirtieron en una herramienta clave para que las empresas se acerquen a los consumidores. Hoy en día estar presentes en las principales redes sociales, es una estrategia de marketing relacional, que se convierte en una oportunidad única de crear contenido de calidad, promocionar y conquistar nuevas audiencias. Mucho se ha dicho de la importancia que tienen actualmente las redes sociales, especialmente con la presencia de influencers y rostros que promocionan marcas y productos. A esto se le llama Contenido Generado por Usuarios o UCG y busca visibilizar la relación entre usuarios y marcas, aunque hoy en día todos podemos ser voceros de una marca; especialmente cuando hoy muchos de los viajes de usuario nacen desde una simple e inocente búsqueda en Google.

Sin embargo es importante entender que dar el paso al mundo digital no se trata de desvanecerse como tienda o negocio físico, todo lo contrario se trata de ofrecer más alternativas a los clientes, facilitándoles los procesos y adaptándose a sus necesidades, Envíos a domicilio, devoluciones o recogidas en la tienda física son alternativas que, bien utilizadas, aportarán un gran valor añadido al negocio.

\section{Conclusiones}

Vender en tiempos de pandemia (COVID-19) significa adaptarse a las preocupaciones de los clientes, al confinamiento y las limitaciones de movilidad, cambios de paradigmas y sobre todo a nuevos hábitos de consumo. Teniendo la capacidad de girar 360 grados y flexibilizar los procesos en los canales de venta y marketing, son algunos tics primordiales que se deben tener para mantenerse a flote en los momentos más complicados y no cerrar las puertas, como le tocó a muchas empresas que no lograron sobrevivir a esta pandemia.

Para utilizar mejor los recursos económicos en las empresas es conveniente implementar el Inbound Marketing, debido a que los recursos se convierten en una inversión y no en un gasto, sobre todo en estos momentos de pandemia, donde deben ser utilizados con responsabilidad.

\section{Referencias bibliográficas}

Cavero, J. (2019, diciembre). Win-Win. Recuperado 19 de octubre de 2020, de Mentor Day WikiTips website: https://mentorday.es/wikitips/win-win/ 
DANE. (2018). Evolución y situación actual de las Mipymes en Colombia. Recuperado 9 de octubre de 2020, de https://www.dinero.com/edicion-impresa/pymes/articulo/evolucion-y-situacion-actual-de-las-mipymesen-colombia/222395

Famanía, I. (2020, mayo 5). 3 FÓRMULAS PARA VENDER MEJOR. Recuperado 19 de octubre de 2020, de https://mdcmagazine.com/articulos/planners-tips/how-to-do/3-formulas-para-vender-mejor

Fernandez, E. (2013). ¿Qué es el Inbound Marketing? Recuperado 9 de octubre de 2020, de INCRENTA - Blog Colombia website: http://increnta.com/co/blog/que-es-el-inbound-marketing/

Gómez, E. (2020). El incalculable valor de las colaboraciones win-win. Recuperado 9 de octubre de 2020, de Think Big website: https://empresas.blogthinkbig.com/colaboraciones-win-win/

Gutzwiller, D., Dunz-Real, C., y Reinsurance, S. (2020). E-business: Una nueva forma de hacer negocios: el negocio electrónico para el mercado latinoamericano.

Halligan, B., y Shah, D. (2009). Inbound Marketing. 256.

Hernández Sampieri, R., y Mendoza Torres, C. P. (2018). Metodología de la investigación: Las rutas cuantitativa, cualitativa y mixta. México: McGraw-Hill Education.

Hill, B. (2020). Marketing During Times of Uncertainty: Advice from Marketing Experts. Recuperado 9 de octubre de 2020, de Metigy website: https://metigy.com/metigy-learning/marketing-during-times-ofuncertainty-advice-from-marketing-experts/

Hugo, V. (2020). SALUD PUBLICA Y COVID-19 reflexiones de una sociedad en crisis.

Kaminsky, G. (2004). Varieties of Currency Crises. Annals of Economics and Finance, 14.

Martínez, T. L. (2000). Técnicas de análisis de datos en investigación de mercados. Pirámide. Recuperado de https://dialnet.unirioja.es/servlet/libro?codigo=4825

Meijomil, S. (2020). Nuevo Estudio del Inbound Marketing: Edición 2020 ya disponible. Recuperado 9 de octubre de 2020, de https://www.inboundcycle.com/blog-de-inbound-marketing/nuevo-estudio-delinbound-marketing-edicion-2020

Morales, A. (2020). Relaciones en tiempos de pandemia: COVID-19 y bienestar animal, ambiental y humano. Revista Facultad Nacional de Agronomía Medellín, 73. https://doi.org/10.15446/rfnam.v73n2.86957

Quelch, J., y Jocz, K. (2009). How to Market in a Downturn'. Harvard business review, 87.

Samsing, C. (2019, diciembre). ¿Qué es Inbound Marketing? Recuperado 19 de octubre de 2020, de https://blog.hubspot.es/marketing/que-es-inbound-marketing-slide-share

Schüler, L. (2020). Descubre la importancia del Marketing Digital en la crisis actual. Recuperado 9 de octubre de 2020, de Blog de Marketing Digital de Resultados | Colombia website: https://www.rdstation.com/co/blog/importancia-marketing-digital-en-la-crisis/

Shelly. (2010). The Importance and Value of B2B Advertising During Times of ... Recuperado de https://www.slideshare.net/Shelly38/the-importance-and-value-of-b2b-advertising-during-times-of

Toledo, A., y Armas, N. (2020). TIPS de marketing en épocas de COVID y Post COVID. CienciAmérica, 9, 99. https://doi.org/10.33210/ca.v9i2.300

Valdés, P. (2019). Inbound Marketing: Qué es, origen, metodología y filosofía. Recuperado 9 de octubre de 2020, de https://www.inboundcycle.com/inbound-marketing-que-es

Esta obra está bajo una Licencia Creative Commons Attribución-NoCommercial 4.0 International

(cc) BY-NC 